\title{
Some Topological Properties of Fuzzy Antinormed Linear Spaces
}

\author{
Ljubiša D. R. Kočinac $\mathbb{D}$ \\ University of Nǐs, Faculty of Sciences and Mathematics, 18000 Niš, Serbia \\ Correspondence should be addressed to Ljubiša D. R. Kočinac; lkocinac@gmail.com \\ Received 17 January 2018; Accepted 9 May 2018; Published 8 August 2018 \\ Academic Editor: Hee S. Kim \\ Copyright (C) 2018 Ljubiša D. R. Kočinac. This is an open access article distributed under the Creative Commons Attribution License, \\ which permits unrestricted use, distribution, and reproduction in any medium, provided the original work is properly cited. \\ The definition of fuzzy antinorm is modified. Some topological properties of finite dimensional fuzzy antinormed linear space are \\ studied. Fuzzy anticonvergence and statistical fuzzy anticonvergence are defined and their properties are studied. We also discuss \\ some boundedness properties in fuzzy antinormed linear spaces.
}

\section{Introduction}

The concept of fuzzy set was introduced by Zadeh [1] in 1965. Thereafter, fuzzy set theory found applications in different areas of mathematics and its applications in other sciences.

The concept of fuzzy norm was introduced by Katsaras [2] in 1984. In 1992, by using fuzzy numbers, Felbin [3] introduced the fuzzy norm on a linear space. Cheng and Mordeson [4] introduced another idea of fuzzy norm on a linear space, and in 2003 Bag and Samanta [5] modified the definition of fuzzy norm of Cheng-Mordeson [4]. In [6] a comparative study of the fuzzy norms defined by Katsaras [2], Felbin [3], and Bag and Samanta [5] was given.

In the paper [6] the idea of fuzzy antinorm was introduced. On the basis of this idea, Jebril and Samanta [7] introduced the concept of fuzzy antinorm on a linear space based on the notion of continuous triangular conorm, first applied in investigation of probabilistic metric spaces [8]. Dinda, Samanta, and Jebril further modified this concept in [9] and also defined fuzzy $\alpha$-anticonvergence. In this paper we use this later approach to investigate statistical versions of anticonvergence. For more information on statistical convergence see [10]. Recall that statistical convergence is defined by using the asymptotic or natural density $\delta(A)$ of a subset $A$ of natural numbers $\mathbb{N}$, defined by $\delta(A)=\lim _{n \rightarrow \infty}(\mid\{k \in A: k \leq$ $n\} \mid / n)$ if this limit exists. $A \subset \mathbb{N}$ is said to be statistically dense if $\delta(A)=1$.

The paper is organized in the following way. After this introductory section, in Section 2 we give basic definitions and preliminary results which we use in the sequel. Section 3 is devoted to fuzzy anticonvergence, and in Section 4 we consider some covering properties in fuzzy antinormed linear spaces.

\section{Preliminaries}

This section contains some basic definition and preliminary results which we need for further exposition.

Definition 1 (see [8]). A binary operation $\diamond:[0,1] \times[0,1] \longrightarrow$ $[0,1]$ is said to be a continuous t-conorm if it satisfies the following conditions:

(i) $\diamond$ is commutative and associative.

(ii) $\diamond$ is continuous.

(iii) $a \diamond 0=a, a \in[0,1]$.

(iv) $a \leq c$ and $b \leq d$ imply $a \diamond b \leq c \diamond d$ for $a, b, c, d \in[0,1]$.

Classical examples of continuous $t$-conorms are $a \diamond b=$ $a+b-a b, a \diamond b=\max \{a, b\}, a \diamond b=\min \{a+b, 1\}$.

We often use idempotent $t$-conorms $\diamond$ (i.e., satisfying $a \diamond$ $a=a$ for each $a \in[0,1])$.

Recall now the notion of fuzzy antinorm in a linear space with respect to a continuous $t$-conorm following [9].

In what follows $E$ will denote a real linear space with the zero element $\theta$ and $\diamond$ will be a continuous $t$-conorm.

Definition 2 (see [9]). Let $E$ be a real linear space and $\diamond$ a $t$ conorm. A fuzzy subset $v: E \times \mathbb{R} \longrightarrow \mathbb{R}$ of $E \times \mathbb{R}$ is called a fuzzy antinorm on $E$ with respect to the $t$-conorm $\diamond$ if, for all $x, y \in E$, 
(FaN1) for each $t \in(-\infty, 0], \nu(x, t)=1$;

(FaN2) for each $t \in(0, \infty), v(x, t)=0$ if and only if $x=\theta$;

(FaN3) for each $t \in(0, \infty), v(c x, t)=v(x, t /|c|)$ if $c \neq 0$

(FaN4) for all $s, t \in \mathbb{R}, v(x+y, s+t) \leq v(x, s) \diamond v(y, t)$;

(FaN5) $\lim _{t \rightarrow \infty} v(x, t)=0$.

Note that if $v$ is the antinorm $v$ in the definition above, then $v(x, t)$ is nonincreasing with respect to $t$ for each $x \in E$.

The following are examples of fuzzy antinorms with respect to a corresponding $t$-conorm and show how a fuzzy antinorm can be obtained from a norm.

Example 3. (1) ([9]) Let $(E,\|\cdot\|)$ be a normed linear space and let the $t$-norm $\diamond$ be given by $a \diamond b=a+b-a b$. Define $\nu: E \times \mathbb{R} \longrightarrow[0,1]$ by

$$
v(x, t)= \begin{cases}0, & \text { if } t>\|x\| ; \\ \frac{\|x\|}{t+\|x\|}, & \text { if } 0<t \leq\|x\| ; \\ 1, & \text { if } t \leq 0 .\end{cases}
$$

Then $v$ is a fuzzy antinorm on $E$ with respect to the $t$-conorm $\diamond$.

(2) $([7])$ Let $(E,\|\cdot\|)$ be a normed linear space and let the $t$ conorm $\diamond$ be given by $a \diamond b=\max \{a, b\}$. Define $\nu: E \times \mathbb{R} \longrightarrow$ $[0,1]$ by

$$
v(x, t)= \begin{cases}\frac{\|x\|}{t+\|x\|}, & \text { if } t>0 \\ 1, & \text { if } t \leq 0 .\end{cases}
$$

Then $v$ is a fuzzy antinorm on $E$ with respect to the $t$-conorm $\diamond$.

Example 4 (see [9]). Let $(E,\|\cdot\|)$ be a normed linear space and let the $t$-conorm $\diamond$ be given by $a \diamond b=a+b-a b$. Define $\nu: E \times \mathbb{R} \longrightarrow[0,1]$ by

$$
\nu(x, t)= \begin{cases}0, & \text { if } t>\|x\| ; \\ 1, & \text { if } t \leq\|x\| .\end{cases}
$$

Then $v$ is a fuzzy antinorm on $E$ with respect to the $t$-conorm $\diamond$. This antinorm $v$ satisfies also the following:

$$
\text { (FaN6) } \nu(x, t)<1 \text { for each } t>0 \text { implies } x=\theta \text {. }
$$

Example 5 (see [9]). Let $(E,\|\cdot\|)$ be a normed linear space and consider the $t$-conorm $\diamond$ defined by $a \diamond b=\min \{a+b, 1\}$. Define $v: E \times \mathbb{R} \longrightarrow[0,1]$ by

$$
v(x, t)= \begin{cases}\frac{\|x\|}{2 t-\|x\|}, & \text { if } t>\|x\| ; \\ 1, & \text { if } t \leq\|x\| .\end{cases}
$$

Then $v$ is a fuzzy antinorm on $E$ with respect to the $t$-norm $\diamond$. Note that this $v$ satisfies the condition ( FaN6) and also the following:
(FaN7) $v(x, \cdot)$ is a continuous function on $\mathbb{R}$ and strictly decreasing on the subset $\{t: 0<v(x, t)<1\}$ of $\mathbb{R}$.

\section{Statistical Fuzzy (Anti)Convergence}

Definition 6. A sequence $\left(x_{n}\right)_{n \in \mathbb{N}}$ in a fuzzy antinormed linear space $(E, \nu, \diamond)$ is said to be $\nu$-convergent to a point $x \in E$ if for each $\varepsilon>0$ and each $t>0$ there is $n_{0} \in \mathbb{N}$ such that

$$
v\left(x_{n}-x, t\right)<\varepsilon \quad \text { for each } n \geq n_{0} .
$$

In this case we write $\left(x_{n}\right) \stackrel{\nu}{\rightarrow} x$.

Definition 7. A sequence $\left(x_{n}\right)_{n \in \mathbb{N}}$ in a fuzzy antinormed linear space $(E, \nu, \diamond)$ is said to be statistically $\nu$-convergent to a point $x \in E$ if for each $\varepsilon>0$ and each $t>0$

$$
\delta\left(\left\{n \in \mathbb{N}: \nu\left(x_{n}-x, t\right)<\varepsilon\right\}\right)=0 .
$$

In this case we write $\left(x_{n}\right) \stackrel{s t-v}{\longrightarrow} x$.

The following theorem is a slight modification of a theorem from [9].

Theorem 8 (see [9]). Let $(E, \nu, \diamond)$ be a fuzzy antinormed linear space with respect to an idempotent $t$-conorm $\diamond$, and let $v$ satisfy (FaN6). Then for each $\lambda \in(0,1)$ the function $\|x\|_{\lambda}: X \longrightarrow[0, \infty)$ defined by

$$
\|x\|_{\lambda}=\bigwedge\{t>0: \nu(x, t) \leq 1-\lambda\}
$$

is a norm on $E$ (called an $\lambda$-norm generated by $\nu$ ), and $\Lambda:=$ $\left\{\|\cdot\|_{\lambda}: \lambda \in(0,1)\right\}$ is an ascending family of norms on $E$.

Convention. We use the notation $(E, \Lambda)$ for the family of normed linear spaces $\left\{\left(E,\|\cdot\|_{\lambda}\right): \lambda \in(0,1)\right\}$ and call $(E, \Lambda)$ also a fuzzy antinormed linear space.

Lemma 9. In a fuzzy antinormed linear space (E, $\Lambda$ ) with respect to an idempotent $t$-conorm $\diamond$ satisfying (FaN6) and (FaN7) a sequence is statistically $\nu$-convergent if and only if it is statistically $\lambda$-convergent for each $\lambda \in(0,1)$.

Proof. $(\Longrightarrow)$ : Let $\left(x_{n}\right)_{n \in \mathbb{N}}$ be a sequence in $E$ such that $\left(x_{n}\right)_{n \in \mathbb{N}} \stackrel{s t-v}{\longrightarrow} x$, i.e., for each $t>0$.

$$
s t-\lim _{n \longrightarrow \infty} v\left(x_{n}-x, t\right)=0 .
$$

Fix $\lambda \in(0,1)$. So, $s t-\lim _{n \rightarrow \infty} \nu\left(x_{n}-x, t\right)=0<1-\lambda$. There exists a statistically dense set $M \subset \mathbb{N}$ so that, for each $n \in M$,

$$
\nu\left(x_{n}-x, t\right)<1-\lambda .
$$

Since $\left\|x_{n}-x\right\|_{\lambda}=\bigwedge\left\{t>0: \nu\left(x_{n}-x, t\right) \leq 1-\lambda\right\}$ we have $\left\|x_{n}-x\right\|_{\lambda} \leq t$ for all $n \in M$. As $t>0$ was arbitrary, for each $\lambda \in(0,1)$, by (FaN6), we have $\left\|x_{n}-x\right\|_{\lambda}$ which statistically converges to 0 . 
$(\Longleftarrow)$ : Suppose now that for each $\lambda \in(0,1),\left\|x_{n}-x\right\|_{\lambda}$ statistically converges to 0 . This means that for each $\lambda \in(0,1)$ and each $\varepsilon>0$ there is a statistically dense $M_{\lambda} \subset \mathbb{N}$ such that

$$
\left\|x_{n}-x\right\|_{\lambda}<\varepsilon
$$

for each $n \in M_{\lambda}$. Therefore,

$$
v\left(x_{n}-x, \varepsilon\right)=\bigwedge\left\{1-\lambda:\left\|x_{n}-x\right\|_{\lambda} \leq \varepsilon\right\}
$$

implies

$$
\nu\left(x_{n}-x, \varepsilon\right) \leq 1-\lambda
$$

for each $\lambda \in(0,1)$ and each $n \in M_{\lambda}$, which means

$$
\nu\left(x_{n}-x, \varepsilon\right) \stackrel{s t-v}{\longrightarrow} 0
$$

that is,

$$
\left(x_{n}\right) \stackrel{s t-v}{\longrightarrow} x
$$

The relations of fuzzy $\lambda$-anticonvergence, fuzzy $\lambda$ anticauchyness, and fuzzy $\lambda$-anticompactness with respect to their corresponding increasing family of norms are studied in the following part of this section.

Definition 10. Let $(E, \Lambda)$ be a fuzzy antinormed linear space and $\lambda \in(0,1)$. A sequence $\left(x_{n}\right)_{n \in \mathbb{N}}$ in $E$ is said to be fuzzy statistically $\lambda$-anticonvergent in $E$ if there exist $x \in E$ and $M \subset$ $\mathbb{N}$ with $\delta(M)=1$ such that, for all $t>0$,

$$
v\left(x_{n}-x, t\right)<1-\lambda \text {. }
$$

In this case we write $\left(x_{n}\right) \stackrel{a-s t-\lambda}{\longrightarrow} x$ and $x$ is called a fuzzy statistical $\lambda$-antilimit of $\left(x_{n}\right)_{n \in \mathbb{N}}$.

Theorem 11. Let $(E, \Lambda)$ be a fuzzy antinormed linear space with respect to an idempotent $t$-conorm $\diamond$ satisfying (FaN6). Then statistical fuzzy $\lambda$-antilimit of a fuzzy statistically $\lambda$-anticonvergent sequence is unique.

Proof. Let $\left(x_{n}\right)_{n \in \mathbb{N}}$ be a fuzzy statistically $\lambda$-anticonvergent sequence converging to distinct points $x$ and $y$ in $E$. This means that for each $t>0$ there are two subsets $M_{x}$ and $M_{y}$ of $\mathbb{N}$ with $\delta\left(M_{x}\right)=1=\delta\left(M_{y}\right)$ such that we have $v\left(x_{n}-x, t / 2\right)<$ $1-\lambda$ for each $n \in M_{x}$ and $\nu\left(x_{n}-y, t / 2\right)<1-\lambda$ for each $n \in M_{y}$. The set $M=M_{x} \cap M_{y}$ is sequentially dense in $\mathbb{N}$, and by the assumption on $\diamond$ for each $n \in M$ we have

$$
\begin{aligned}
\nu(x-y, t) & =v\left(x-x_{n}+x_{n}-y, t\right) \\
& \leq v\left(x_{n}-x, \frac{t}{2}\right) \diamond \nu\left(x_{n}-y, \frac{t}{2}\right) \\
& <(1-\lambda) \diamond(1-\lambda)=1-\lambda
\end{aligned}
$$

Therefore, $v(x-y, t)<1$ for each $t>0$. By (FaN6) one obtains $x-y=\theta$, i.e., $x=y$.
Theorem 12. Let $(E, \Lambda)$ be a fuzzy antinormed linear space with respect to an idempotent $t$-conorm $\diamond$ satisfying (FaN6). Then:

(1) If $\left(x_{n}\right) \stackrel{a-s t-\lambda}{\longrightarrow} x$ and $\left(y_{n}\right) \stackrel{a-s t-\lambda}{\longrightarrow} y$, then $\left(x_{n}+\right.$ $\left.y_{n}\right) \stackrel{a-s t-\lambda}{\longrightarrow} x+y$;

(2) If $\left(x_{n}\right) \stackrel{a-s t-\lambda}{\longrightarrow} x$ and $r \in \mathbb{R}$, then $\left(r \cdot x_{n}\right) \stackrel{a-s t-\lambda}{\longrightarrow} r x$.

Proof. (1) Since $\left(x_{n}\right) \stackrel{a-s t-\lambda}{\longrightarrow} x$ for each $t>0$ there is $M_{1} \subset \mathbb{N}$ with $\delta\left(M_{1}\right)=1$ such that $\nu\left(x_{n}-x, t / 2\right)<1-\lambda$ for each $n \in M_{1}$. Similarly, from $\left(y_{n}\right) \stackrel{a-s t-\lambda}{\longrightarrow} z$ it follows that for each $t>0$ there is a set $M_{2} \subset \mathbb{N}$ with $\delta\left(M_{2}\right)=1$ such that $\nu\left(y_{n}-\right.$ $y, t / 2)<1-\lambda$ for each $n \in M_{2}$. Then $M=M_{1} \cap M_{2}$ is such that $\delta(M)=1$ and for each $t>0$ and each $n \in M$ we have

$$
\begin{aligned}
\nu\left(x_{n}+y_{n}-x-y, t\right) \leq & \nu\left(x_{n}-x, \frac{t}{2}\right) \\
& \diamond \nu\left(y_{n}-y, \frac{t}{2}\right) \\
& <(1-\lambda) \diamond(1-\lambda)=1-\lambda,
\end{aligned}
$$

which means $\left(x_{n}+y_{n}\right) \stackrel{a-s t-\lambda}{\longrightarrow} x+y$.

(2) The fact $\left(x_{n}\right) \stackrel{a-s t-\lambda}{\longrightarrow} x$ implies that for each $t>0$ there is a statistically dense subset $M$ of $\mathbb{N}$ such that $\nu\left(x_{n}-y, t\right)<$ $1-\lambda$ for each $n \in M$. Then for each $t>0$

$$
v\left(r x_{n}-r x, t\right)=v\left(x_{n}-x, \frac{t}{|r|}\right)<1-\lambda
$$

for each $n \in M$; i.e., $\left(r \cdot x_{n}\right) \stackrel{a-s t-\lambda}{\longrightarrow} r x$.

Theorem 13. Let $(E, \Lambda)$ be a fuzzy antinormed linear space with respect to an idempotent $t$-conorm $\diamond$. If $\left(x_{n}\right)_{n \in \mathbb{N}}$ is a fuzzy statistically $\lambda$-anticonvergent sequence in $(E, \Lambda)$ statistically converging to $x \in E$, then $\left\|x_{n}-x\right\|_{\lambda}$ statistically converges to 0 .

Proof. By assumption there is a set $M \subset \mathbb{N}$ with $\delta(M)=1$ such that for each $t>0$ and each $n \in M$ we have $\nu\left(x_{n}-x, t\right)<$ $1-\lambda$. In other words, $\left\|x_{n}-x\right\|_{\lambda}<t$ for each $n \in M$. Since $t$ was arbitrary we have that $\left\|x_{n}-x\right\|_{\lambda}$ statistically converges to 0 .

Definition 14. Let $\lambda \in(0,1)$. A sequence $\left(x_{n}\right)_{n \in \mathbb{N}}$ in a fuzzy antinormed linear space $(E, \Lambda)$ (with respect to a $t$-conorm $\diamond)$ is said to be fuzzy statistically $\lambda$-anti-Cauchy if for every $t>0$ there is a set $M \subset \mathbb{N}$ such that $\delta(M)=1$ and for all $m, n \in M, v\left(x_{n}-x_{m}, t\right)<1-\lambda$.

Theorem 15. Let $(E, \Lambda)$ be a fuzzy antinormed linear space with respect to an idempotent $t$-conorm $\diamond$ satisfying (FaN6) and $\lambda \in(0,1)$. Then every fuzzy statistically $\lambda$-anticonvergent sequence $\left(x_{n}\right)_{n \in \mathbb{N}}$ in $(E, \Lambda, \diamond)$ is fuzzy statistically $\lambda$-antiCauchy.

Proof. Since $\left(x_{n}\right)_{n \in \mathbb{N}}$ is fuzzy statistically $\lambda$-anticonvergent to some $x \in E$, for each $t>0$, there is a set $M \subset \mathbb{N}$ with $\delta(M)=1$ 
such that $v\left(x_{n}-x, t / 2\right)<1-\lambda$ for each $n \in M$. Then for all $m, n \in M$ we have

$$
\begin{aligned}
\nu\left(x_{n}-x_{m}, t\right) & =v\left(x_{n}-x+x-x_{m}, t\right) \\
& \leq v\left(x_{n}-x, \frac{t}{2}\right) \diamond \nu\left(x_{m}-x, \frac{t}{2}\right) \\
& <(1-\lambda) \diamond(1-\lambda)=1-\lambda
\end{aligned}
$$

which means that $\left(x_{n}\right)_{n \in \mathbb{N}}$ is fuzzy statistically $\lambda$-anti-quasiCauchy in $(E, \Lambda)$.

Theorem 16. Let $(E, \Lambda)$ be a fuzzy antinormed linear space with respect to an idempotent $t$-conorm $\diamond$. Then every statistically Cauchy sequence $\left(x_{n}\right)_{n \in \mathbb{N}}$ in $\left(E,\|\cdot\|_{\lambda}\right), \lambda \in(0,1)$, is fuzzy statistically $\lambda$-anti-quasi-Cauchy in $(E, \Lambda)$.

Proof. Let $\lambda \in(0,1)$ be arbitrary and fixed. Since $\left(x_{n}\right)_{n \in \mathbb{N}}$ is a statistical Cauchy sequence in $\left(E,\|\cdot\|_{\lambda}\right)$, for any $\varepsilon>0$ there is a set $M \subset \mathbb{N}$ with $\delta(M)=1$ such that for all $m, n \in M$ we have $\left\|x_{n}-x_{m}\right\|_{\lambda}<\varepsilon$. It means that for each $m, n \in M$

$$
\bigwedge\left\{t>0: \nu\left(x_{n}-x_{m}, t\right) \leq 1-\lambda\right\}<\varepsilon
$$

which implies the existence of $t_{0}<\varepsilon$ such that $\nu\left(x_{n}-x_{m}, t_{0}\right)<$ $1-\lambda$. It follows that $\nu\left(x_{n}-x_{m}, \varepsilon\right)<1-\lambda$, and as $\varepsilon$ was arbitrary, we conclude that $\nu\left(x_{n}-x_{m}, t\right)=1-\lambda$ for each $t>0$ and all $m, n \in M$. This means that $\left(x_{n}\right)_{n \in \mathbb{N}}$ is fuzzy statistically $\lambda$-anti-quasi-Cauchy sequence in $(E, \Lambda)$. But, $\lambda$ also was an arbitrary element in $(0,1)$ so that we have that $\left(x_{n}\right)_{n \in \mathbb{N}}$ is fuzzy statistically $\lambda$-anti-quasi-Cauchy in $(E, \Lambda)$ for each $\lambda \in(0,1)$.

Definition 17. A fuzzy antinormed linear space $(E, \Lambda)$ (with respect to a $t$-conorm $\diamond)$ is said to be fuzzy statistically $\lambda$ anticomplete, $\lambda \in(0,1)$, if every fuzzy statistically $\lambda$-antiCauchy sequence in $E$ fuzzy statistically $\lambda$-anticonverges in E.

Theorem 18. Let $(E, \Lambda)$ be a fuzzy antinormed linear space with respect to an idempotent $t$-conorm $\diamond$. If $(E, \Lambda)$ is fuzzy statistically $\lambda$-anticomplete, then $E$ is statistically complete with respect to $\|\cdot\|_{\lambda}$ for each $\lambda \in(0,1)$.

Proof. Let $\lambda \in(0,1)$ be fixed and let $\left(x_{n}\right)_{n \in \mathbb{N}}$ be a statistically Cauchy sequence in $E$ with respect to $\|\cdot\|_{\lambda}$. By the previous theorem $\left(x_{n}\right)_{n \in \mathbb{N}}$ is fuzzy statistically $\lambda$-anti-Cauchy in $(E, \Lambda)$. Therefore, there is $x \in E$ and a subset $M$ of $\mathbb{N}$ with $\delta(M)=1$ such that, for each $t>0$ and each $n \in M, v\left(x_{n}-x, t\right)<1-\lambda$. By Theorem 13 , this means $\left\|x_{n}-x\right\|_{\lambda}$ statistically converges to 0 ; i.e., $\left(x_{n}\right)_{n \in \mathbb{N}}$ statistically converges to $x$ with respect to $\|\cdot\|_{\lambda}$. Therefore, $\left(E,\|\cdot\|_{\lambda}\right)$ is statistically complete.

\section{Some Covering Properties}

Let $(E, \nu, \diamond)$ be a fuzzy antinormed linear space where $\diamond$ is idempotent. Given $x \in E, \varepsilon \in(0,1)$, and $t>0$, the set

$$
B_{\nu}(x, \varepsilon, t):=\{y \in E: \nu(y-x, t)<\varepsilon\}
$$

is called the open ball with center $x$ and radius $\varepsilon$ with respect to $t$.

For each point $y \in B_{\gamma}(x, \varepsilon, t)$ there is an open ball with center $y$ contained in $B_{\gamma}(x, \varepsilon, t)$. Let $\nu(y-x, t)=\alpha<\varepsilon$ and set $\beta=\varepsilon-\alpha$. We prove $B_{v}(y, \beta, t / 2) \subset B_{\gamma}(x, \varepsilon, t)$. Let $p \in$ $B_{v}(y, \beta, t / 2)$. Then $\nu(p-y, t / 2)<\beta$ so that we have

$$
\begin{aligned}
\nu(p-x, t) & =v\left(p-y+y-x, \frac{t}{2}+\frac{t}{2}\right) \\
& =v\left(p-y, \frac{t}{2}\right) \diamond \nu\left(y-p, \frac{t}{2}\right) \\
& <(\varepsilon-\alpha) \diamond \varepsilon \leq \varepsilon \diamond \varepsilon=\varepsilon,
\end{aligned}
$$

i.e., $p \in B_{v}(x, \varepsilon, t)$.

Therefore, the collection

$$
\{B(x, \varepsilon, t): x \in E, \varepsilon \in(0,1), t>0\}
$$

is a base of a topology on $E$; denote this topology by $\tau_{v}$. Notice that the collection

$$
\left\{B\left(x, \frac{1}{n}, t\right): x \in E, n \in \mathbb{N}, t>0\right\}
$$

is also a base for $\tau_{v}$. The topology $\tau_{v}$ is Hausdorff and first countable.

The following definitions are motivated by definitions of the classical Menger, Rothberger, and Hurewicz covering properties (for details see the papers [11-14]).

Recall that a topological space has the Menger (Rothberger, Hurewicz) covering property if for each sequence $\left(\mathcal{U}_{n}: n \in \mathbb{N}\right)$ of open covers of $X$ there is a sequence $\left(\mathscr{V}_{n}: n \in \mathbb{N}\right)$ (resp., $\left.\left(U_{n}: n \in \mathbb{N}\right),\left(\mathscr{W}_{n}: n \in \mathbb{N}\right)\right)$ such that, for each $n \in \mathbb{N}, \mathscr{V}_{n}$ is a finite subset of $\mathscr{U}_{n}$ (resp., $U_{n} \in \mathscr{U}_{n}, \mathscr{W}_{n}$ is a finite subset of $\mathscr{U}_{n}$ ) and $X=\bigcup_{n \in \mathbb{N}} \bigcup \mathscr{V}_{n}$ (resp., $X=\bigcup_{n \in \mathbb{N}} U_{n}$, each $x \in X$ belongs to $\cup \mathscr{W}_{n}$ for all but finitely many $n$ ).

Definition 19. A fuzzy antinormed linear space $(E, \nu, \diamond)$ is said to be

\section{M: Menger-bounded (or M-bounded), \\ R: Rothberger-bounded (or R-bounded), \\ $\mathrm{H}$ : Hurewicz-bounded (or $\mathrm{H}$-bounded)}

if for each sequence $\left(\varepsilon_{n}: n \in \mathbb{N}\right)$ of elements of $(0,1)$ and each $t>0$ there is a sequence

M: $\left(A_{n}: n \in \mathbb{N}\right)$ of finite subsets of $E$ such that $E=$ $\bigcup_{n \in \mathbb{N}} \bigcup_{a \in A_{n}} B\left(a, \varepsilon_{n}, t\right)$

R: $\left(x_{n}: n \in \mathbb{N}\right)$ of elements of $E$ such that $E=$ $\bigcup_{n \in \mathbb{N}} B\left(x_{n}, \varepsilon_{n}, t\right)$,

$\mathrm{H}:\left(A_{n}: n \in \mathbb{N}\right)$ of finite subsets of $E$ such that for each $x \in E$ there is $n_{0} \in \mathbb{N}$ such that $x \in \bigcup_{a \in A_{n}} B\left(a, \varepsilon_{n}, t\right)$ for all $n \geq n_{0}$.

A fuzzy antinormed linear space $(E, \nu, \diamond)$ is said to be precompact (respectively, pre-Lindelöf) if for every $\varepsilon \in(0,1)$ and every $t>0$ there is a finite (respectively, countable) set $A \subset E$ such that $E=\bigcup_{a \in A} B_{\gamma}(a, \varepsilon, t)$. 
Evidently,

$$
\begin{aligned}
\text { precompact } & \Longrightarrow \mathrm{H} \text { - bounded } \Longrightarrow \mathrm{M} \text { - bounded } \\
& \Longrightarrow \text { pre - Lindelöf, } \\
\mathrm{R} \text { - bounded } & \Longrightarrow \mathrm{M} \text { - bounded. }
\end{aligned}
$$

Example 20. Let $(E,\|\cdot\|)$ be a normed linear space with the Menger (Rothberger, Hurewicz) property. Consider the fuzzy antinormed linear space $(E, \nu, \diamond)$, where $\nu$ and $\diamond$ are as in Example 3(1). Then this fuzzy antinormed linear space is $\mathbf{M}$ bounded (R-bounded, H-bounded).

Consider only the M-bounded case because the other two are shown quite similarly.

Let $\left(\varepsilon_{n}: n \in \mathbb{N}\right)$ be a sequence in $(0,1)$ and let $t>0$. As $(E,\|\cdot\|)$ has the Menger covering property, there is a sequence $\left(A_{n}: n \in \mathbb{N}\right)$ of finite subsets of $X$ such that

$$
E=\bigcup_{n \in \mathbb{N}} \bigcup_{a \in A_{n}} K\left(a, \varepsilon_{n}\right),
$$

where $K(a, \varepsilon)=\{y \in X:\|a-y\|<\varepsilon\}$.

Let $x \in X$. There is $n \in \mathbb{N}$ and a point $a_{n} \in A_{n}$ satisfying $\left\|x-a_{n}\right\|<\varepsilon_{n}$. Then

$$
\begin{aligned}
& v\left(x-a_{n}, t\right) \\
& \quad= \begin{cases}0, & \text { if } t>\left\|x-a_{n}\right\| ; \\
\frac{\left\|x-a_{n}\right\|}{t+\left\|x-a_{n}\right\|}, & \text { if } t \leq\left\|x-a_{n}\right\|, t>0 ;\end{cases}
\end{aligned}
$$

If $t>\left\|x-a_{n}\right\|$, then $v\left(x-a_{n}, t\right)=0$ so that $x \in B_{\nu}\left(a_{n}, \varepsilon_{n}, t\right)$. If $t>0$ and $t \leq\left\|x-a_{n}\right\|$, then $v\left(x-a_{n}, t\right)=\left(\left\|x-a_{n}\right\| /(t+\| x-\right.$ $\left.\left.a_{n} \|\right)\right) \varepsilon_{n}$; i.e., in this case also $x \in B_{\nu}\left(a_{n}, \varepsilon_{n}, t\right)$.

Therefore, $E=\bigcup_{n \in \mathbb{N}} \bigcup_{a \in A_{n}} B_{\nu}\left(a, \varepsilon_{n}, t\right)$; i.e., $(E, \nu, \diamond)$ is $\mathbf{M}$ bounded.

Example 21. Let $E=\mathbb{R}$ and $\|\cdot\|=|\cdot|$, and let $\diamond$ be defined as in Examples 3(1) and 20. Then the fuzzy antinormed space $(E, \nu, \diamond)$ is $\mathrm{M}$-bounded by the previous example and the fact that $(\mathbb{R},|\cdot|)$ has the Menger property $[12,13]$. On the other hand, $(E, v, \diamond)$ is not R-bounded.

Indeed, if we take the sequence $\left(2^{-n}: n \in \mathbb{N}\right) \subset(0,1)$ and $t=2^{-1}$, then $X$ cannot be covered by the open balls $B\left(x_{n}, 2^{-n}, 2^{-1}\right)$ for any choice of elements $x_{n}, n \in \mathbb{N}$, from $E$. Otherwise, we would have that for every $x \in \mathbb{R}$ the following holds:

$$
\frac{\left|x-x_{n}\right|}{2^{-1}+\left|x-x_{n}\right|}<2^{-n},
$$

which means

$$
\mathbb{R}=\bigcup_{n \in \mathbb{N}}\left(x_{n}-2^{-n}, x_{n}+2^{-n}\right) .
$$

However, it is impossible.

We end with the following result on M-boundedness.

If $(E, \nu, \diamond)$ is a fuzzy antinormed space and $S \subset E$, then $\left(S, v_{S}, \diamond\right)$, where $v_{S}=v \uparrow(S \times \mathbb{R})$, is also a fuzzy antinormed space and it is called the fuzzy antinormed subspace of $(X, v, \diamond)$.
Theorem 22. Every fuzzy antinormed subspace $\left(S, v_{S}, \diamond\right)$ of an $\mathrm{M}$-bounded ( $\mathrm{H}$-bounded) fuzzy antinormed space $(E, v, \diamond)$ is also $\mathrm{M}$-bounded ( $\mathrm{H}$-bounded).

Proof. Consider the M-bounded case; the proof for $\mathrm{H}$ boundedness is quite similar. Let $\left(\varepsilon_{n}: n \in \mathbb{N}\right)$ be a sequence of elements of $(0,1)$ and $t>0$. Continuity of $\diamond$ implies that for each $n \in \mathbb{N}$ there is $\delta_{n} \in(0,1)$ such that $\delta_{n} \diamond \delta_{n}<\varepsilon_{n}$.

For each $n \in \mathbb{N}$ let

$$
\begin{aligned}
& Q_{n} \\
& =\left\{q \in P_{n}: \text { there is } y \in S \text { with } y \in B_{\nu}\left(q, \delta_{n}, \frac{t}{2}\right)\right\} .
\end{aligned}
$$

Further, for each $q \in Q_{n}$ choose an element $y_{q} \in S$ such that $y_{q} \in B_{\gamma}\left(q, \delta_{n}, t / 2\right)$ and set

$$
A_{n}=\left\{y_{q}: q \in Q_{n}\right\}
$$

Let us show that the sequence $\left(A_{n}: n \in \mathbb{N}\right)$ of finite subsets of $S$ witnesses for $\left(\varepsilon_{n}: n \in \mathbb{N}\right)$ and $t$ that $\left(S, v_{S}, \diamond\right)$ is $\mathrm{M}$ bounded.

Let $z \in S$ be an arbitrary element. There are $n \in \mathbb{N}$ and $p \in P_{n}$ such that $z \in B_{\nu}\left(p, \delta_{n}, t / 2\right)$. By the definition of $Q_{n}$ it follows that $p \in Q_{n}$ so that there is $z_{p} \in A_{n}$ such that $z_{p} \in$ $B_{\nu}\left(p, \delta_{n}, t / 2\right)$; hence $p \in B_{\nu}\left(z_{p}, \delta_{n}, t / 2\right)$. So, we have

$$
v\left(p-z_{p}, \frac{t}{2}\right)<\delta_{n}
$$

By (FaN4) we have

$$
\begin{aligned}
v\left(z-z_{p}, t\right) & \leq v\left(z-p, \frac{t}{2}\right) \diamond \nu\left(p, z_{p}, \frac{t}{2}\right)<\delta_{n} \diamond \delta_{n} \\
& <\varepsilon_{n},
\end{aligned}
$$

which means $z \in B_{\nu}\left(z_{p}, \varepsilon_{n}, t\right)$. As $z \in S$ was arbitrary and $z_{p} \in A_{n}$ we conclude

$$
S=\bigcup_{n \in \mathbb{N}} \bigcup_{a \in A_{n}} B_{v}\left(a, \varepsilon_{n}, t\right)
$$

i.e., $S$ is M-bounded.

\section{Data Availability}

No data were used to support this study.

\section{Conflicts of Interest}

The author declares that there are no conflicts of interest regarding the publication of this paper.

\section{References}

[1] L. A. Zadeh, "Fuzzy sets," Information and Control, vol. 8, no. 3, pp. 338-353, 1965.

[2] A. K. Katsaras, "Fuzzy topological vector spaces. II," Fuzzy Sets and Systems, vol. 12, no. 2, pp. 143-154, 1984. 
[3] C. Felbin, "The completion of a fuzzy normed linear space," Journal of Mathematical Analysis and Applications, vol. 174, no. 2, pp. 428-440, 1993.

[4] S. C. Cheng and J. N. Mordeson, "Fuzzy linear operators and fuzzy normed linear spaces," Bulletin of the Calcutta Mathematical Society, vol. 86, no. 5, pp. 429-436, 1994.

[5] T. Bag and S. K. Samanta, "Finite dimensional fuzzy normed linear spaces," Journal of Fuzzy Mathematics, vol. 11, no. 3, pp. 687-705, 2003.

[6] T. Bag and S. K. Samanta, "A comparative study of fuzzy norms on a linear space," Fuzzy Sets and Systems, vol. 159, no. 6, pp. 670-684, 2008.

[7] I. H. Jebril and T. K. Samanta, "Fuzzy anti-normed linear space," Journal of Mathematics and Technology, pp. 66-77, 2010.

[8] B. Schweizer and A. Sklar, "Statistical metric spaces," Pacific Journal of Mathematics, vol. 10, pp. 313-334, 1960.

[9] B. Dinda, T. K. Samanta, and I. H. Jebril, "Fuzzy anti-norm and fuzzy $\alpha$-anti-convergence," Demonstratio Mathematica, vol. 45, no. 4, pp. 739-754, 2012.

[10] G. Di Maio and L. D. R. Kočinac, "Statistical convergence in topology," Topology and its Applications, vol. 156, no. 1, pp. 2845, 2008.

[11] L. D. R. Kočinac, "Selection principles in uniform spaces," Note di Matematica, vol. 22, no. 2, pp. 127-139, 2003/04.

[12] L. D. R. Kočinac, "Selected results on selection principles," in Proceedings of the Third Seminar on Geometry and Topology, pp. 71-104, Tabriz, Iran, July 2004.

[13] L. D. R. Kočinac, "Some covering properties in topological and uniform spaces," Proceedings of the Steklov Institute of Mathematics, vol. 252, no. 1, pp. 122-137, 2006.

[14] L. D. R. Kočinac, "Selection properties in fuzzy metric spaces," Filomat, vol. 26, no. 2, pp. 305-312, 2012. 


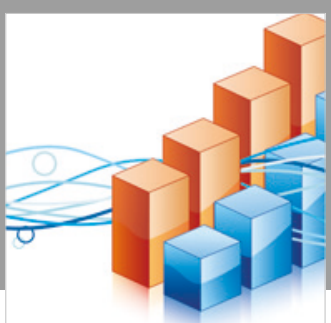

Advances in

Operations Research

\section{-n-m}
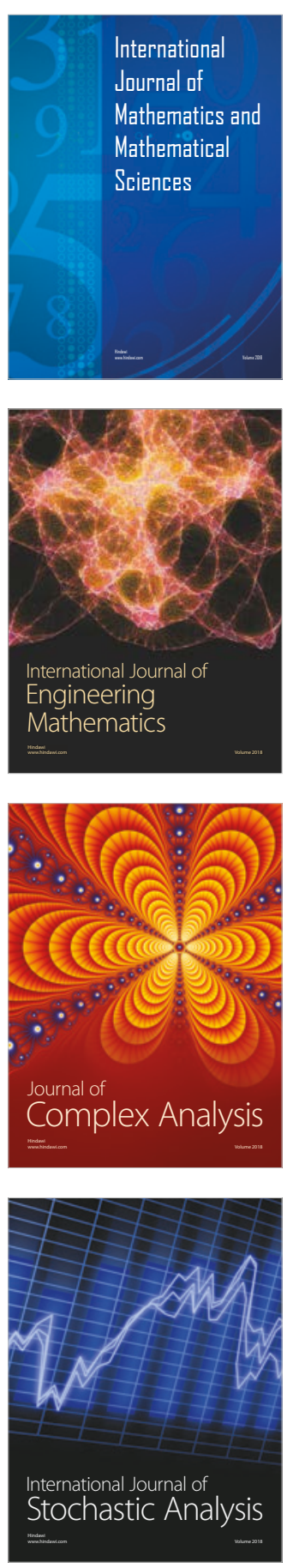
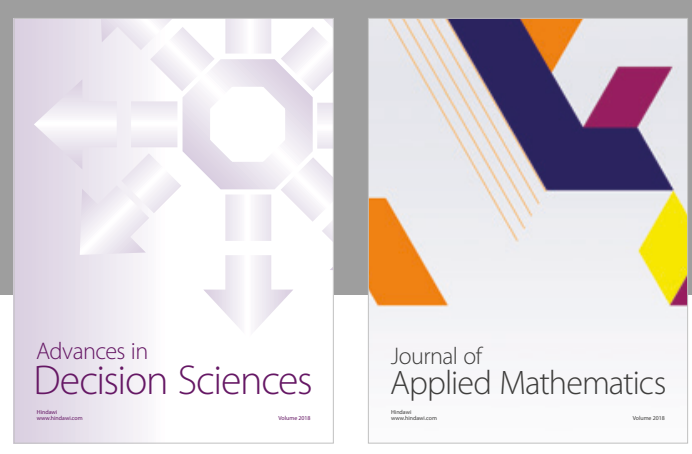

Journal of

Applied Mathematics
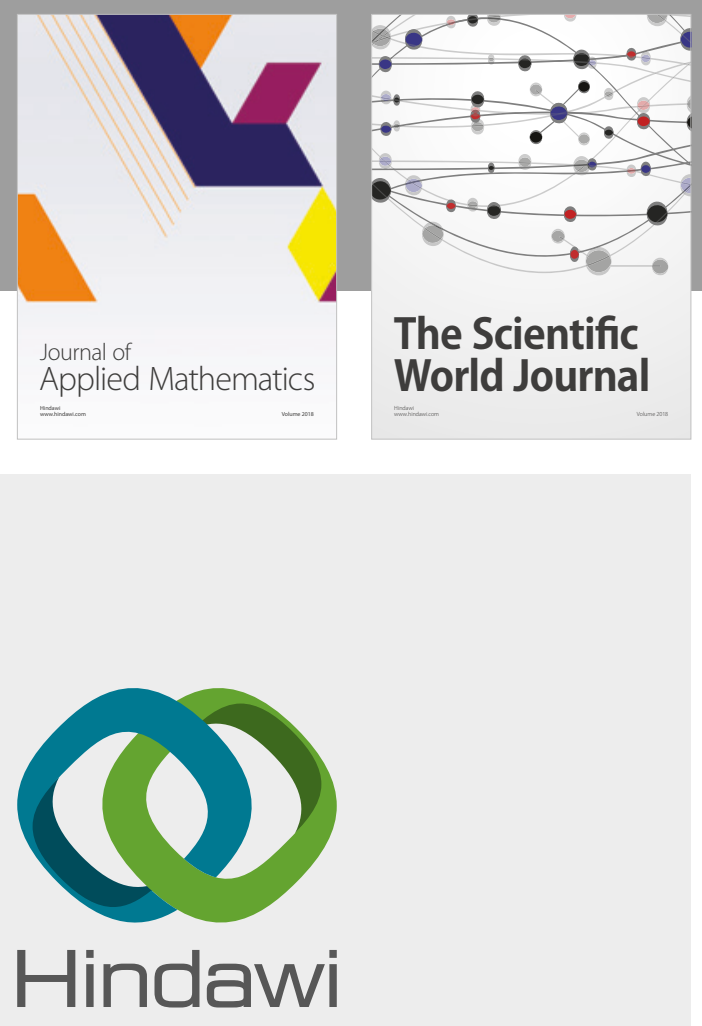

Submit your manuscripts at

www.hindawi.com

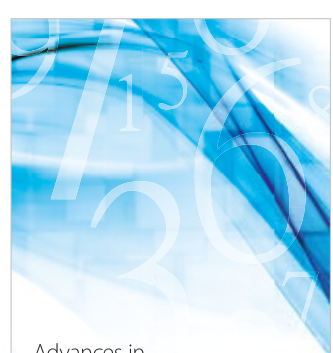

Advances in
Numerical Analysis
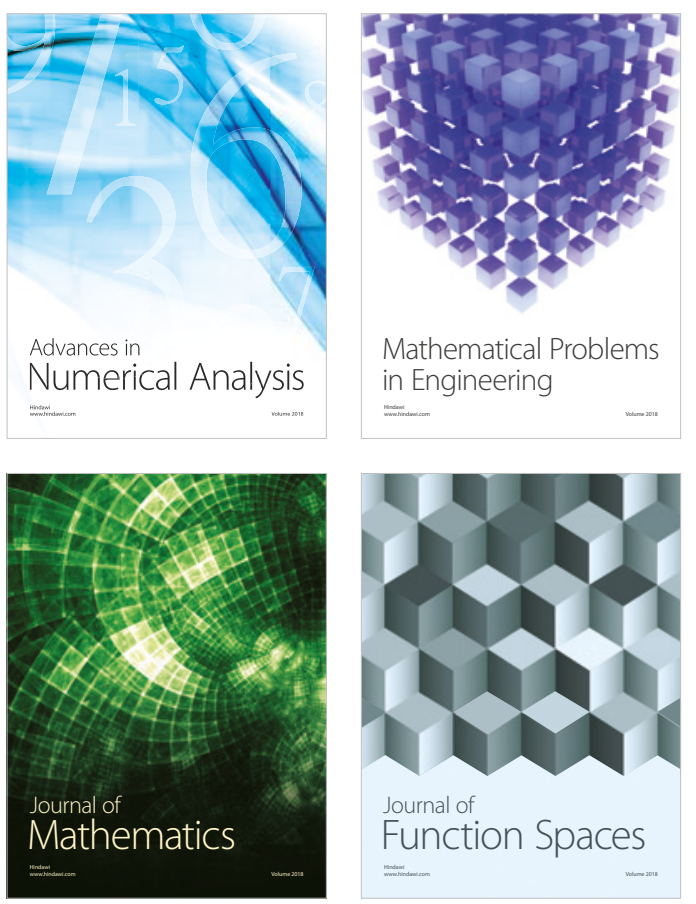

Mathematical Problems in Engineering

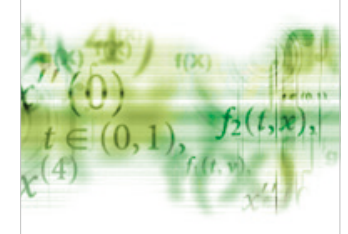

International Journal of

Differential Equations

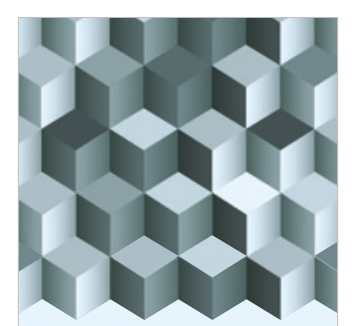

Journal of

Function Spaces

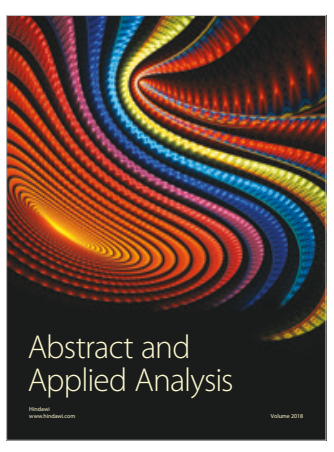

The Scientific

World Journal

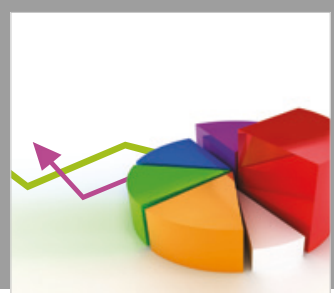

Journal of

Probability and Statistics
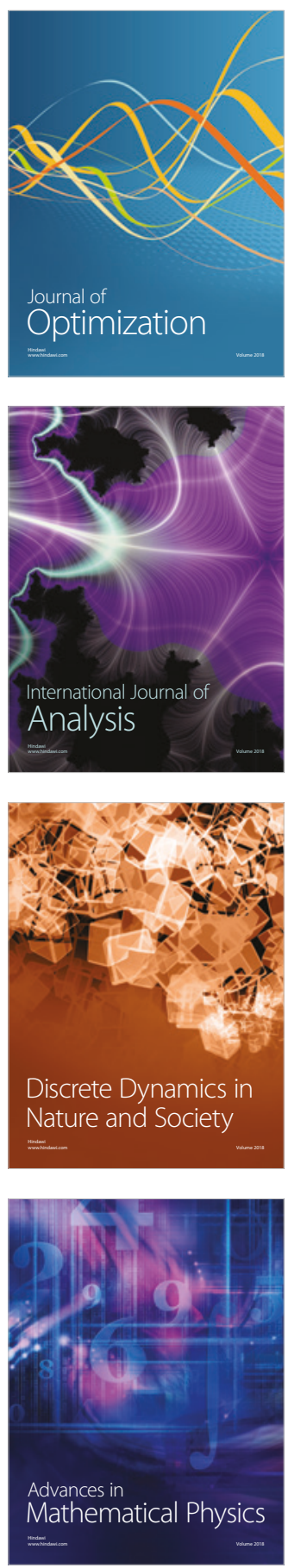